59

\section{EFFECT OF INSULIN ON THE METABOLIC AND NEUROENDOCRINE STRESS RESPONSES TO CRITICAL ILLNESS IN CHILDREN}

R.G. Branco1 ${ }^{1}$, G.S. Conrado², F. Cabral'2, C. Korb², R. Camargo ${ }^{2}$, E. Jarmola², P.C.R. Garcia², J.P. Piva ${ }^{2}$, R.C. Tasker ${ }^{1}$

${ }^{1}$ Paediatrics, Cambridge University, Cambridge, UK, ${ }^{2}$ Paediatric Intensive Care Unit, Hospital Sao Lucas da PUCRS, Porto Alegre, Brazil

Background/aims: Neuroimmunoendocrine and metabolic dysfunctions are present in critical illness. We investigated whether insulin could reverse or limit these changes.

Methods: Open-label randomized controlled physiology study of the effects of insulin during critical illness. 31 children with multiple organ dysfunction syndrome were recruited: 13children received continuous insulin infusion $(0.1 \mathrm{Unit} / \mathrm{Kg} /$ $\mathrm{hr}$ ) and glucose was added if required to maintain glucose levels between 4.5 and $7.0 \mathrm{mmol} / \mathrm{L}$. Blood samples for neuroimmunoendocrine and metabolic evaluation were obtained before randomization, and 24 and 72 hours later. A growth hormone $(\mathrm{GH})$ oscillation profile was obtained during the first night.

Results: At baseline both groups were similar. There was also no absolute difference in between the groups, at any time, in GH, IGF-1 and IGFBP-3. $\mathrm{GH}$ had higher relative increase in children receiving insulin, which suggests worsened $\mathrm{GH}$ resistance. By day 2, insulin decreased IGFBP-1 levels. At the same time insulin decreased free fatty acid levels, which was accompanied by increased LDL cholesterol and relative increase in HDL levels. Total cholesterol and triglycerides were unchanged. TNF-alpha and interleukin(IL)-1beta decreased in both groups, whereas IL-6 levels were significantly reduced significantly in children receiving insulin.

Conclusions: Insulin does not reverse most of the neuroendocrine changes induced by stress of critical illness. However, it does appear to improve lipid metabolism (preserved LDL and HDL cholesterol) and down-regulate inflammation (decreased IL6 ). These findings suggest that insulin in critical illness acts as a modulator of lipid dysfunction, which suggests new targets for future investigations.
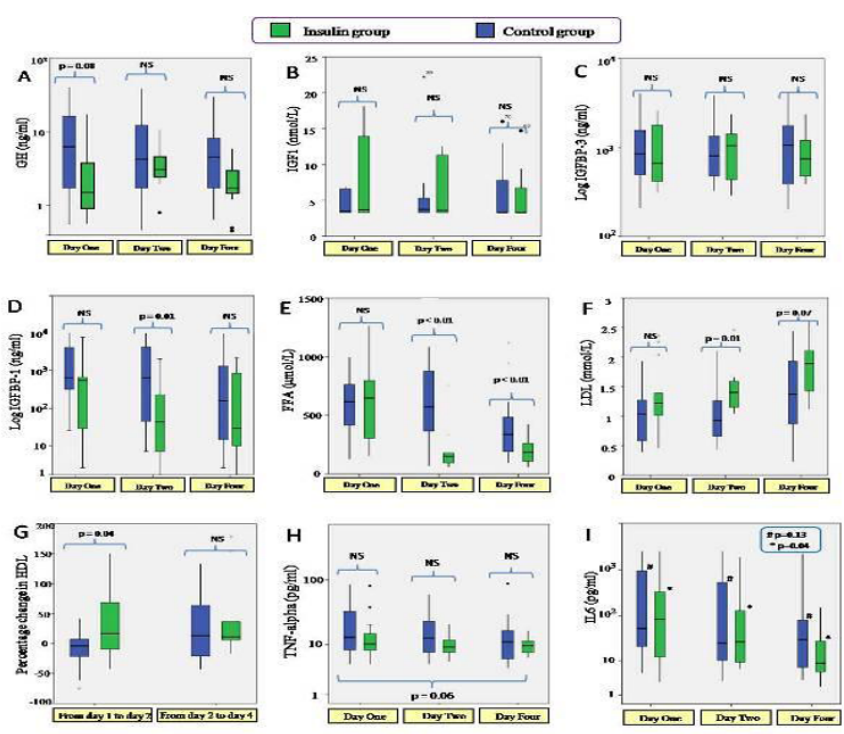

Figure 1. Insulin effect on neuroendocrine, metabolic and inflammatory stress responses. There was no absolute differcnee in GH, IGF-1 and IFGBP-3 levels betwocn groups at any time (A,
B, C), but insulin decreased IGFBP-1 levels on day 2 (D). Insulin also decreased FFA (E) lovels, which was aceompanied by an increase in LDL cholestcrol (F) and a perontage reduced signifieantly only in children receiving

[Figure 1]

60

\section{INTENSIVE INSULIN THERAPY AND BRIEF HYPOGLYCEMIA DO NOT INCREASE MARKERS OF NEUROLOGICAL INJURY IN CRITICALLY ILL CHILDREN}

\author{
M. Gielen, I. Vanhorebeek, M. Boussemaere, \\ P.J. Wouters, D. Mesotten, G. Van den Berghe \\ Intensive Care Medicine, K.U.Leuven, Leuven, \\ Belgium
}

Background and aims: Targeting normoglycemia with intensive insulin therapy (IIT) improved shortterm outcome of pediatric intensive care unit (PICU) patients, but concomitantly increased the incidence of hypoglycemia. Both hyperglycemia and hypoglycemia may adversely affect the developing brain in children. We studied the impact of targeting normoglycemia with IIT on circulating markers of brain injury in critically ill children.

Methods: This was a pre-planned analysis of critically ill pediatric patients included in a randomized controlled study on IIT. Patients were assigned to IIT targeting normal-for-age fasting blood glucose levels throughout intensive care or insulin infusion only to prevent excessive hyperglycemia. Serum S100B and neuron-specific enolase (NSE), biomarkers of astrocytic and neuronal damage, respectively, were measured on fixed days $(n=700)$ and in a "nested case-control" design before and after hypoglycemia $(n=126)$. 